
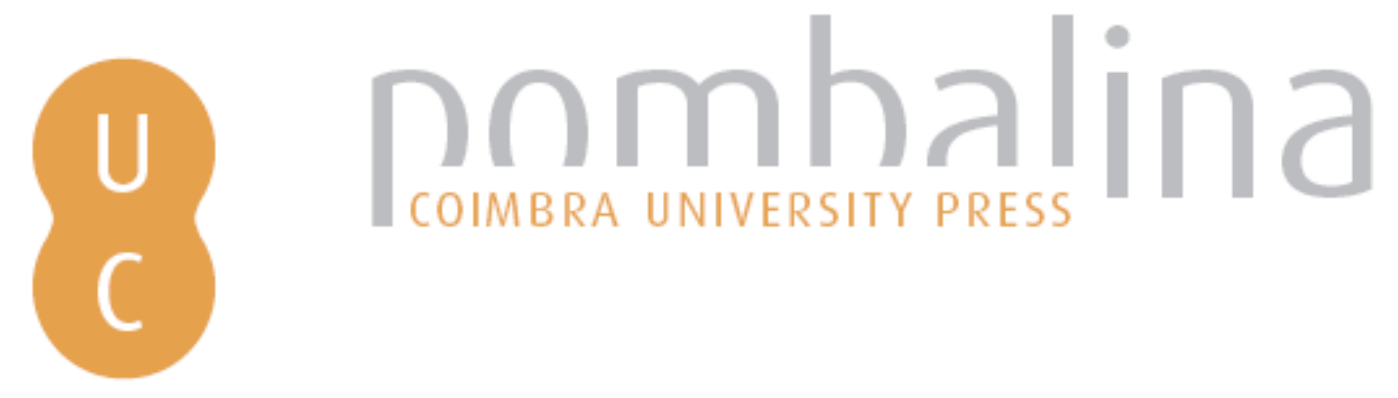

\title{
La ciudad como espacio de riesgo ambiental: estimación de la contaminación del aire y de la población expuesta en metrópolis españolas
}

\author{
Autor(es): $\quad$ Cañada Torrecilla, Rosa; Moreno Jiménez, Antonio; Martínez Suárez, \\ Pedro; Vidal Domínguez, María Jesús \\ Publicado por: Imprensa da Universidade de Coimbra; RISCOS - Associação \\ Portuguesa de Riscos, Prevenção e Segurança \\ URL \\ persistente: \\ URI:http://hdl.handle.net/10316.2/34884 \\ DOI: \\ DOI:http://dx.doi.org/10.14195/978-989-96253-3-4_83 \\ Accessed : $\quad$ 26-Apr-2023 11:41:43
}

A navegação consulta e descarregamento dos títulos inseridos nas Bibliotecas Digitais UC Digitalis, UC Pombalina e UC Impactum, pressupõem a aceitação plena e sem reservas dos Termos e Condições de Uso destas Bibliotecas Digitais, disponíveis em https://digitalis.uc.pt/pt-pt/termos.

Conforme exposto nos referidos Termos e Condições de Uso, o descarregamento de títulos de acesso restrito requer uma licença válida de autorização devendo o utilizador aceder ao(s) documento(s) a partir de um endereço de IP da instituição detentora da supramencionada licença.

Ao utilizador é apenas permitido o descarregamento para uso pessoal, pelo que o emprego do(s) título(s) descarregado(s) para outro fim, designadamente comercial, carece de autorização do respetivo autor ou editor da obra.

Na medida em que todas as obras da UC Digitalis se encontram protegidas pelo Código do Direito de Autor e Direitos Conexos e demais legislação aplicável, toda a cópia, parcial ou total, deste documento, nos casos em que é legalmente admitida, deverá conter ou fazer-se acompanhar por este aviso.

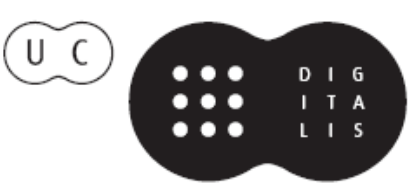



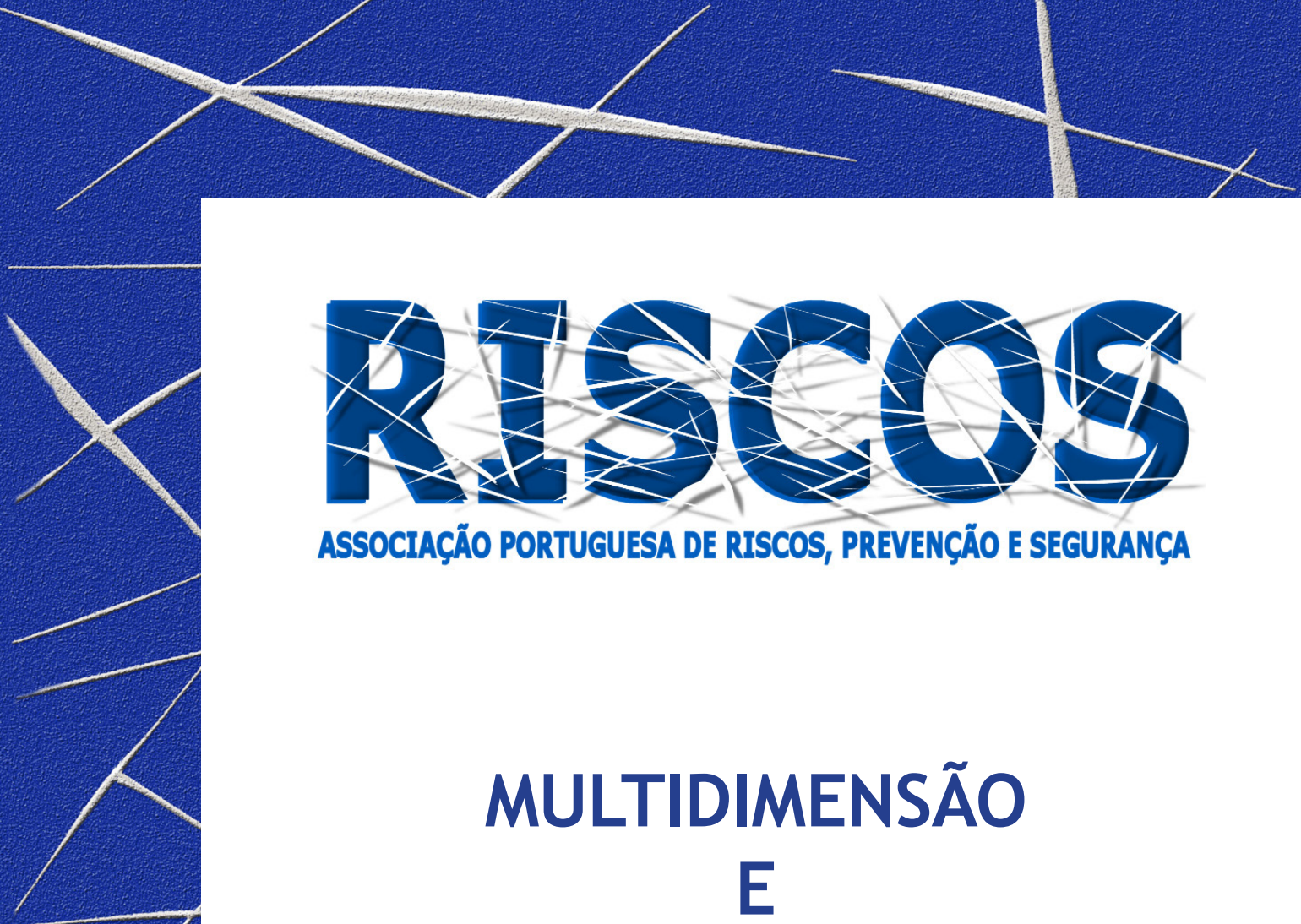

ASSOCIAÇÃO PORTUGUESA DE RISCOS, PREVENCCÃO E SEGURANÇA

MULTIDIMENSÃO

E
TERRITÓRIOS DE RISCO

III Congresso Internacional

I Simpósio Ibero-Americano

VIII Encontro Nacional de Riscos

Guimarães

2014 


\title{
LA CIUDAD COMO ESPACIO DE RIESGO AMBIENTAL: ESTIMACIÓN DE LA CONTAMINACIÓN DEL AIRE Y DE LA POBLACIÓN EXPUESTA EN METRÓPOLIS ESPAÑOLAS
}

\author{
Rosa Cañada Torrecilla \\ Departamento de Geografía, Facultad de Filosofía y Letras. Universidad Autónoma de Madrid \\ rosa.canada@uam.es \\ Antonio Moreno Jiménez \\ Departamento de Geografía, Facultad de Filosofía y Letras. Universidad Autónoma de Madrid \\ antonio.moreno@uam.es \\ Pedro Martínez Suárez \\ Departamento de Geografía, Facultad de Filosofía y Letras. Universidad Autónoma de Madrid \\ pedro.martínez@uam.e \\ María Jesús Vidal Domínguez \\ Departamento de Geografía, Facultad de Filosofía y Letras. Universidad Autónoma de Madrid \\ mariajesus.vidal@uam.es
}

\begin{abstract}
RESUMEN
La ciudad es un espacio de riesgo ambiental, pues las actividades allí realizadas reducen la calidad del aire al generar contaminantes peligrosos para la salud de las personas y el entorno. Diversos informes de la OMS muestran la relación entre la exposición prolongada a $\mathrm{PM}_{10}, \mathrm{O}_{3}$ y NO 2 y el riesgo de desarrollar enfermedades cardiovasculares, respiratorias y cáncer de pulmón. Los objetivos serán, en primer lugar, estimar los niveles de concentración atmosférica de estos contaminantes en tres ciudades españolas, mediante el empleo de técnicas de interpolación espacial y SIG, y en segundo lugar, determinar cuáles de ellos constituyen una amenaza para la población urbana, qué zonas y qué población vive en zonas con alta contaminación.

Palabras clave: contaminación urbana, interpolación espacial, exposición, población, SIG.
\end{abstract}

\section{Introducción}

La OMS considera que la contaminación atmosférica es el mayor riesgo medioambiental sobre la salud, estima que causa 2 millones de muertes prematuras al año (Gurjar et al., 2010). De los tres contaminantes que abordamos en este estudio, $\mathrm{PM}_{10}, \mathrm{O}_{3}$ y $\mathrm{NO}_{2}$, el primero afecta a las personas más que cualquier otro. Una exposición prolongada a sus componentes (mezcla de partículas sólidas y líquidas, de sustancias orgánicas e inorgánicas suspendidas en el aire) contribuye al riesgo de desarrollar enfermedades cardiovasculares, respiratorias y cáncer de pulmón (WHO, 2013). Estudios realizados en la UE indican que la esperanza de vida se puede reducir 8,6 meses (Gurjar et al., 2010). En el caso del ozono troposférico, contaminante secundario formado por una reacción fotoquímica con los óxidos de nitrógeno, compuestos orgánicos volátiles (COV), metano y monóxido de carbono, excesivas concentraciones, pueden afectar a la salud humana, a los materiales y a la vegetación. Pueden inducir a problemas respiratorios, desencadenar asma y causar problemas pulmonares (Amann et al., 2008, WHO, 2013). Estudios epidemiológicos han demostrado que los síntomas de niños con bronquitis asmáticas aumentan con exposiciones muy largas al $\mathrm{NO}_{2}$, cuya principal fuente de emisión procede de los procesos de combustión llevados a cabo en actividades relacionadas con la generación de calor, generación eléctrica, vehículos a motor, etc. (Gurjar et al., 2010; WHO, 2013).

Considerando lo expuesto, se pretende aquí estudiar la dimensión espacial del riesgo de contaminación por $\mathrm{NO}_{2}, \mathrm{PM}_{10}$ y $\mathrm{O}_{3}$, que con frecuencia superan los umbrales establecidos por la 
legislación europea y por la OMS, en tres grandes ciudades españolas, y medir el área y población amenazadas por ellos.

\section{Datos y métodos}

Las ciudades elegidas para este trabajo son Madrid (3.215.633 hab.), Barcelona (1.611.822 hab.) y Sevilla (700.169 hab.). El ámbito de estudio, el área urbana poblada, excluye los espacios no residenciales y los periféricos no urbanizados, tras una labor de identificación apoyada en el Corine Land Cover 2006 y en imágenes aéreas recientes.

Los datos de contaminación proceden de estaciones oficiales sitas en el interior de los municipios examinados y de otros limítrofes, para mejorar la cobertura. Las variables ambientales seleccionadas han sido la concentración media anual de $\mathrm{NO}_{2}$, de partículas en suspensión inferiores a $10 \mu \mathrm{m}\left(\mathrm{PM}_{10}\right)$ en 2010, y para el ozono $\left(\mathrm{O}_{3}\right)$ se ha utilizado el número de días con valor octohorario superior a $120 \mu \mathrm{g} / \mathrm{m}^{3}$ como promedio de tres años (2010, 2011 y 2012). Los niveles admisibles por la legislación europea y la OMS (tabla I) permitirán establecer las zonas de riesgo de alta contaminación en la ciudad.

Tabla I. Niveles de polución del aire admisibles por la legislación europea y la OMS

\begin{tabular}{|l|l|l|l|}
\hline CONTAMINANTE & PERIODO DE REFERENCIA & $\begin{array}{l}\text { VALOR LÍMITE (UE) } \\
\text { PARA LA SALUD HUMANA }\end{array}$ & VALOR GUÍA OMS \\
\hline $\mathrm{NO}_{2}$ & 1 año & $40 \mu \mathrm{g} / \mathrm{m}^{3}$ & $40 \mu \mathrm{g} / \mathrm{m}^{3}$ \\
\hline $\mathrm{PM}_{10}$ & 1 año & $40 \mu \mathrm{g} / \mathrm{m}^{3}$ & $20 \mu \mathrm{g} / \mathrm{m}^{3}$ \\
\hline $\mathrm{O}_{3}$ & 8 Horas & $\begin{array}{l}120 \mu \mathrm{g} / \mathrm{m}^{3} \text { no más de } 25 \text { días } / \text { año } \\
\text { (promedio 3 años) }\end{array}$ & $100 \mu \mathrm{g} / \mathrm{m}^{3}$ \\
\hline
\end{tabular}

Para disponer de información continua de cada contaminante en el conjunto del espacio urbano se ha recurrido a técnicas de predicción espacial, práctica muy habitual en estudios de variables ambientales, usando Geostatistical Analyst de ArcGIS 10.1.

El procedimiento adoptado constó de varias etapas (véase Cañada et al. (2014), siendo la fase crucial del análisis la aplicación de los dos métodos de interpolación, IDW y Kriging ordinario, cuya diferencia radica en el procedimiento de cálculo de los pesos asignados a los valores muestrales. Una descripción más detallada de estas técnicas se pueden encontrar en Cañada, 2007a,b; Krivoruchko, 2011. Se han seleccionado aquellos métodos que consiguen los menores errores en la predicción: una media de los errores próxima a 0 (mean error), un error cuadrático medio más bajo (root mean square error, RMSE) y un root mean square standarized error (RMSSE) próximo a 1 . Una vez obtenida la capa estimada, se convirtió a raster con una resolución de 50 metros y se "recortó" para ajustarla a la zona urbana poblada.

\section{Análisis de los resultados}

Interpolación espacial del $\mathrm{NO}_{2}$, y estimación de superficie y población expuesta El método de interpolación óptimo para las tres ciudades fue el IDW anisotrópico. La vista de los mapas interpolados denotan que las mayores concentraciones se corresponden con grandes avenidas, con tráfico muy intenso. Por su parte, los niveles admisibles, siempre están vinculados a espacios verdes, con una localización eminentemente periférica (figura 1). 

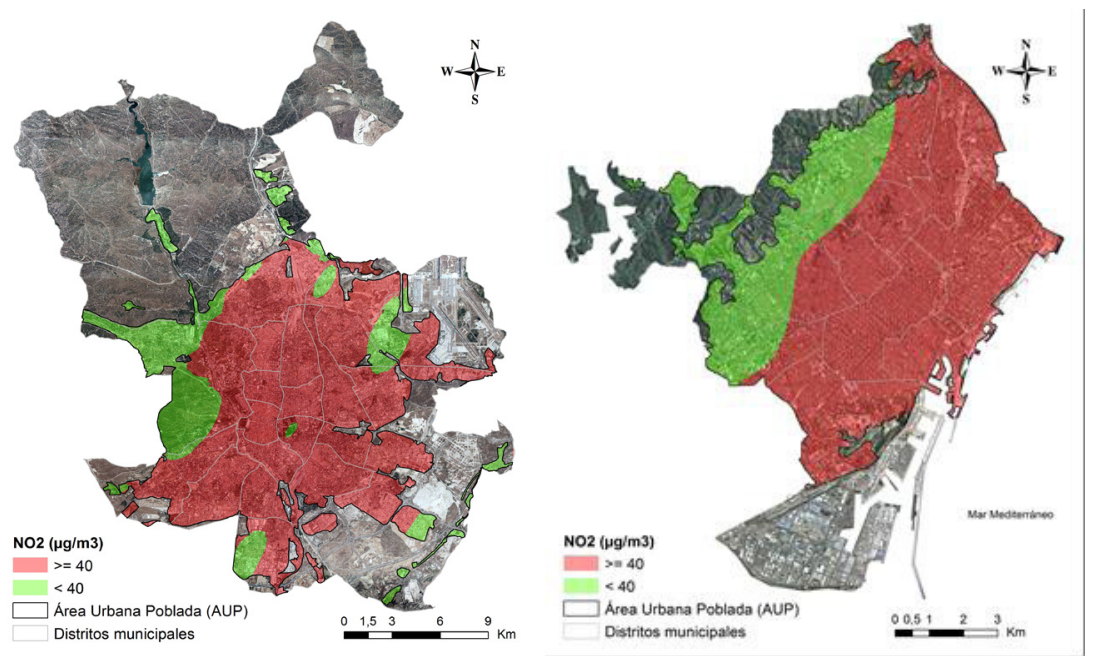

Figura 1. Concentración media anual de $\mathrm{NO}_{2}$ en Madrid y Barcelona, 2010
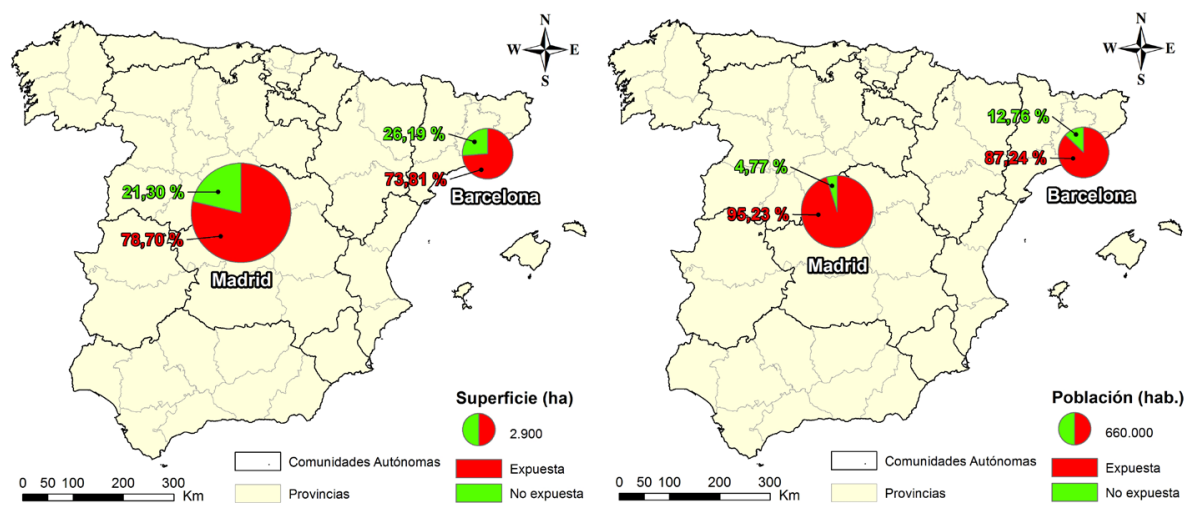

Figura 2. Superficie y población expuesta a $\mathrm{NO}_{2}$ alto en Madrid y Barcelona, 2010.

El 78,7\% de la superficie urbana poblada de Madrid y el 73,81\% de la de Barcelona sufren alta contaminación por $\mathrm{NO}_{2}$, siendo los porcentajes de población expuesta del 95,2\% y del 87,2\% respectivamente (figura 2). En Sevilla no se supera el umbral crítico.

\section{Interpolación espacial del ozono y estimación de superficie y población expuesta}

Los métodos de interpolación del $\mathrm{O}_{3}$ han sido el Kriging anisotrópico en Madrid y el IDW anisotrópico en Barcelona y Sevilla. Factores geográficos, meteorológicos y reacciones fotoquímicas explicarían su distribución espacial. En ciudades con elevadas concentraciones de óxidos de nitrógeno, éstos destruyen el $\mathrm{O}_{3}$, contribuyendo a su disminución en la atmósfera, por el contrario el ozono permenece en aquellos ambientes donde las emisiones a las atmósfera de NOx son menores. Este hecho queda muy bien reflejado en Madrid donde el área expuesta apenas representa el $2 \%$ (zona suburbana del norte y noreste), mientras que en Sevilla alcanza el $58 \%$ (figuras 3 y 4 ). 
Interpolación espacial de $\mathrm{PM}_{10}$ y estimación de superficie y población expuesta

Los métodos que han proporcionado mejor bondad de ajuste han sido el Kriging anisotrópico para Madrid y Barcelona y el IDW anisotrópico en Sevilla. La disposición espacial resultante de las $\mathrm{PM}_{10}$ obedece a cuales son las principales fuentes de emisión: la combustión de carburantes fósiles generada por el tráfico y las zonas con pobre cobertura vegetal o suelos desnudos y secos, más fácilmente erosionables y expuestos a la acción del viento.
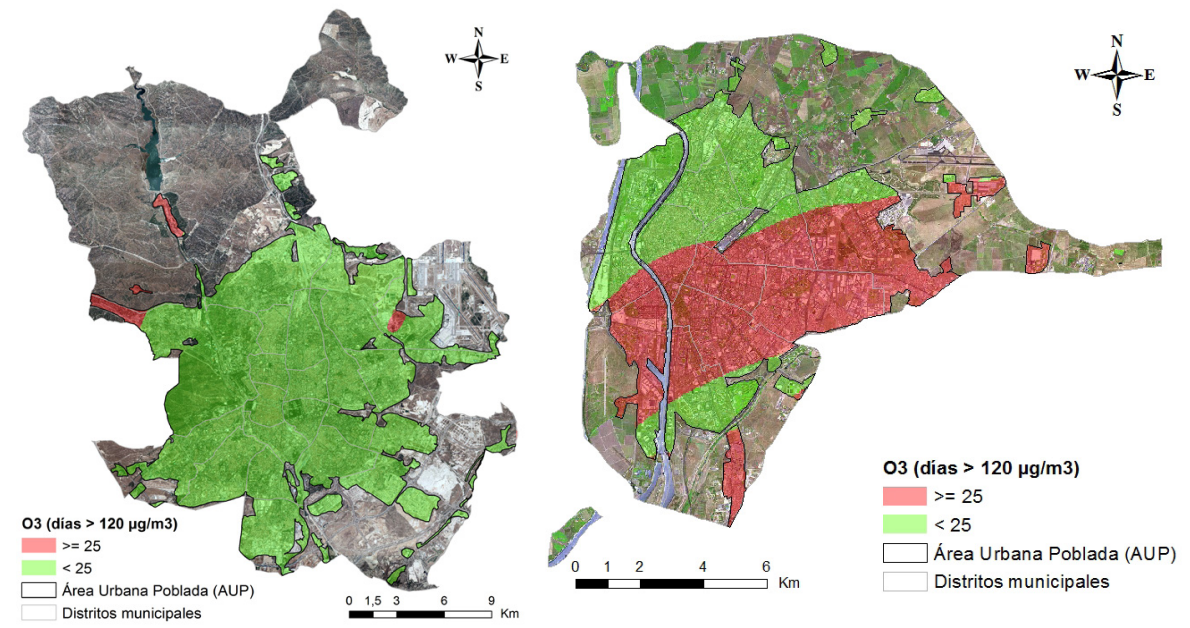

Figura 3. Concentración media anual de Ozono en Madrid y Sevilla, 2010
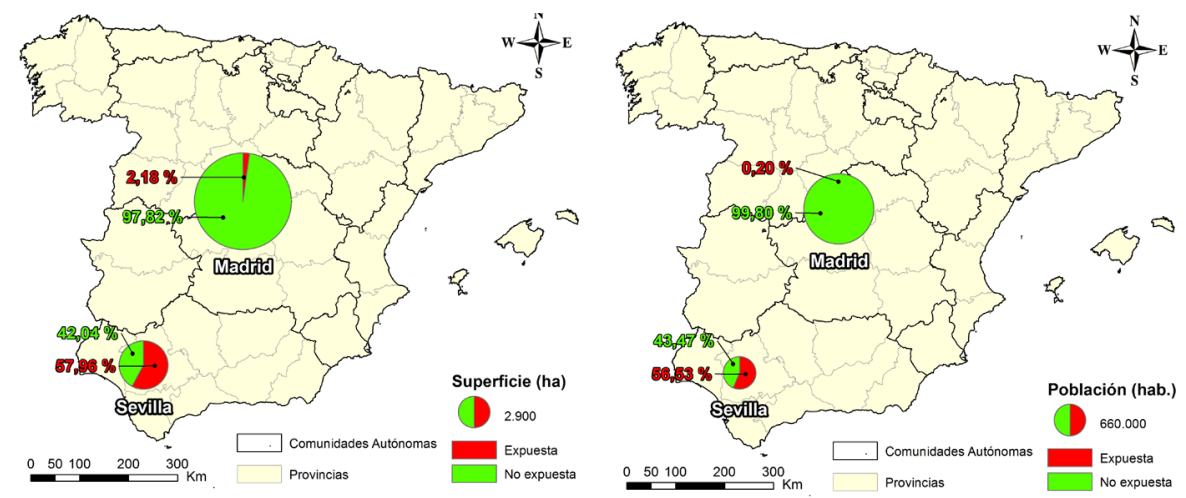

Figura 4. Superficie y población expuesta a $\mathrm{O}_{3}$ alto en Madrid y Sevilla, 2010

Las tres ciudades no tendrían riesgo de contaminación por $\mathrm{PM}_{10}$, si se considera el umbral de 40 $\mu \mathrm{g} / \mathrm{m}^{3}$ establecido por la legislación europea; sin embargo estudios epidemiológicos recogidos por la WHO (2013) ponen de manifiesto los beneficios de una exposición a menores concentraciones en la atmósfera de $\mathrm{PM}_{10}$ y por debajo del umbral establecido por la UE. Por esta razón se ha investigado qué pasaría si se considera espacio de riesgo aquel que supera los 20 $\mu \mathrm{g} / \mathrm{m}^{3}$, umbral aconsejado por la OMS. Los resultados indican que toda el área de Barcelona sufriría de riesgo por $\mathrm{PM}_{10}$ elevado, en Sevilla se alcanzaría el 99,8\% y en Madrid el 91,5\%. 


\section{Conclusiones}

Las ciudades españolas de mayor población constituyen espacios de riesgo de contaminación por $\mathrm{NO}_{2}, \mathrm{PM}_{10} \mathrm{y}_{3}$. Para su estudio, dada la escasa cobertura espacial de las estaciones de medición, se ha recurrido a técnicas de interpolación espacial, estimando así la contaminación en todo el espacio urbano poblado. Los riesgos varían según ciudades y contaminantes. En Madrid y Barcelona hay peligro por $\mathrm{NO}_{2}$ alto. Madrid, y sobre todo Sevilla, constituyen espacios de riesgo de contminación por $\mathrm{O}_{3}$ y ninguna de las tres ciudades sufren amenaza de contaminación por $\mathrm{PM}_{10}$ excesivo.

Los resultados avalan la necesidad de que políticos, planificadores y ciudadanos se conciencien de la deficiente calidad del aire urbano e impulsen medidas más estrictas y acordes con las recomendaciones de la OMS, encaminadas a mejorar los estándares ambientales y la calidad de vida de la población.

\section{Bibliografía}

Amann, M., Derwent, M.D., Forsberg, B., Hänninen, O., Hurley, F., Krzyzanowski, M., de Leeuw, F., J. Liu, S., Mandin, C., Schneider, J., Schwarze, P. y Simpson, D. (2008)- Health risks of ozone from long-range transboundary air pollution. Copenhague, WHO, Regional Office for Europe, $111 \mathrm{p}$.

Cañada Torrecilla, R. (2007a)- Técnicas de interpolación espacial deterministas y exactas: media ponderada por el inverso de la distancia y funciones de base radial”, en Moreno A. (Coord.): Sistemas y análisis de la información geográfica. Madrid, Ra-Ma, p. 781-809.

Cañada Torrecilla, R. (2007b)- Técnicas de interpolación geoestadísticas: Kriging ordinario, en Moreno, A. (Coord.): Sistemas y análisis de la información geográfica. Madrid, Ra-Ma, p. 823-853.

Cañada, R., Moreno, A. y González, H. (2014)- Modelado de la calidad del aire urbano Un experimento metodológico con técnicas de interpolación espacial. Boletín de la Asociación de Geógrafos Españoles, $n^{\circ} 65$, p. 317-342.

Gurjar, B.R, Molina, L.T. y Ojha, C.S.P. (Eds.)(2010)- Air Polution. Health and Environmental Impacts. New York, CRS Press. Taylor and Francis, 518 pp.

Krivoruchko, K. (2011)- Spatial Statistical Data Analysis for GIS Users, Esri Press.

WHO (2013)- Review of evidence on health aspects of air polution-REVIAAP. Copenhagen. Regional Office for Europe, $22 \mathrm{p}$. 